\title{
Cativeiro de aves como fonte de Cryptococcus neoformans na cidade de Campo Grande, Mato Grosso do Sul, Brasil
}

\author{
Avian habitats as sources of Cryptococcus neoformans in the city \\ of Campo Grande, Mato Grosso do Sul, Brazil \\ Wander Fernando de Oliveira Filiú ${ }^{1,2}$, Bodo Wanke ${ }^{3}$, Sandra Maura Agüena ${ }^{1,2}$, \\ Valter Oshiro Vilela ${ }^{1,2}$, Regina Célia Lima Macedo ${ }^{3}$ e Márcia Lazéra ${ }^{3}$
}

\begin{abstract}
Resumo Cryptococcus neoformans é a levedura capsulada causadora de criptococose em humanos e animais. A variedade neoformans, encontrada em diversas fontes ambientais, inclusive habitats de aves, é importante causa de mortalidade em indivíduos com AIDS em todo o Mundo. Contudo, ainda não há estudos sobre a sua ecologia na região Centro Oeste brasileira, onde há registro da ocorrência de casos humanos da micose. Para estudar fontes saprofíticas de C. neoformans, na cidade de Campo Grande, foram coletadas 20 amostras de excretas de aves em distintos ambientes. Suspensão das amostras em salina estéril foram semeadas em placas com meio ágar níger. Após 5 dias, colônias mucóides marrom-escuro foram subcultivadas para identificação através de provas morfofisiológicas, determinação da variedade e sorotipagem. C. neoformans var. neoformans sorotipo $A$ foi isolado de $10(50 \%)$ das amostras, comprovando a ocorrência saprofítica de C. neoformans na cidade de Campo Grande, relacionada a habitat de aves em cativeiro.
\end{abstract}

Palavras-chaves: Cryptococcus neoformans. Aves cativas. Fontes saprofíticas. Criptococose.

\begin{abstract}
Cryptococcus neoformans is an encapsulated yeast agent of human and animal cryptococcosis. The variety neoformans is an important cause of mortality in AIDS-patients throughout the world. It has already been isolated worldwide from different environmental sources, including avian habitats. However, in spite of the occurrence of human cases of cryptococcosis, there are no studies on the ecology of this agent in the Brazilian Central-Western region. To study saprophytic sources of $\mathrm{C}$. neoformans in the city of Campo Grande, 20 samples of avian droppings from distinct environments within the city were collected. The samples were suspended in sterile saline and then smeared on niger seed agar medium. Five days later smooth dark-brown colonies were subcultivated for identification by morphophysiologic tests. The variety and serotype was determined. C. neoformans var. neoformans serotype $A$ was isolated from 10 (50\%) of the samples collected. Consequently, the saprophytic presence of $\mathrm{C}$. neoformans is related to avian habitats.
\end{abstract}

Key-words: Cryptococcus neoformans. Captive birds. Saprophytic sources. Cryptococcosis.

Cryptococcus neoformans, forma assexuada do basidiomiceto Filobasidiella neoformans, é levedura encapsulada uni ou bibrotante de grande importância em micologia médica por ser agente da criptococose. C. neoformans apresenta os sorotipos A, B, C, D e AD distribuídos em duas variedades que diferem em aspectos bioquímicos, ecológicos, antigênicos e genéticos ${ }^{89}$. A variedade neoformans, com os sorotipos $\mathrm{A}, \mathrm{AD}$ e $\mathrm{D}^{89}$, tem distribuição cosmopolita relacionada a solos contaminados naturalmente com excretas de aves 567252627 . Esta variedade tem a capacidade de colonizar a mucosa do papo de pombos, sem causar doença, comportando-se como endosaprófita natural destas aves. A universalidade e a peculiar adaptação de pombos a centros urbanos relacionam-se com a ubiqüidade deste agente fúngico, sendo facilmente isolado de fontes ambientais, inclusive de poeira domiciliar ${ }^{17}{ }^{18}$.

Mais recentemente, foi demonstrada no Brasil a associação saprofítica de C. neoformans var. neoformans com madeira em decomposição em ocos de árvores vivas ${ }^{1011} 1213$, constituindo um novo habitat natural e indicando um possível nicho ecológico primário para o fungo ${ }^{10}$. Sem dúvida, há necessidade de desenvolver estudos similares em outras partes do mundo 2124.

\footnotetext{
1. Laboratório de Análises Clínicas do Hospital Universitário da Universidade Federal de Mato Grosso do Sul, Campo Grande, MS. 2. Laboratório de Análises Clínicas da Universidade para o Desenvolvimento do Estado e da Região do Pantanal, Campo Grande, MS. 3. Serviço de Micologia da Fundação Oswaldo Cruz, Rio de Janeiro, RJ.

Endereço para correspondência: Dr. Wander Fernando de Oliveira Filiú. Rua Pernambuco, 946/802, Monte Castelo, $79010-900$ Campo Grande, MS. e-mail: filiu@ zaz.com.br
}

Recebido para publicação em 27/9/2001. 
C. neoformans var neoformans predomina como agente oportunista ${ }^{28}$, atingindo hospedeiros com deficiência da imunidade celular, ganhando grande importância nos dias atuais, não só pelo aumento populacional mas sobretudo pelo crescente número de hospedeiros suscetíveis: (síndrome da imunodeficiência adquirida) SIDA, leucêmicos, transplantados, portadores de tumores sólidos e pacientes em uso prolongados de corticóides e antibióticos ${ }^{16} 22$.

A infecção por $C$. neoformans var. neoformans é adquirida através da inalação de propágulos de origem ambiental ${ }^{15}$, representados por leveduras desidratadas, menores que $2 \mathrm{~mm}$ de diâmetro, facilmente aerossolizadas. Sugeriu-se que basidiósporos da forma sexuada do fungo, medindo $1,8 \times 2,5 \mathrm{~mm}$, mais resistentes à dessecação, mais facilmente aerossolizados e comprovadamente patogênicos para animais de laboratório, também possam ser inalados ${ }^{31}$.

A variedade gattii, com sorotipos B e C, ocorre em regiões tropicais e subtropicais e comporta-se como patógeno primário, acometendo hospedeiros imunocompetentes $^{923}$. Foi inicialmente associada a Eucalyptus camaldulensis na Austrália ${ }^{3419}$, seguindose seu isolamento de restos vegetais de diversas espécies de eucaliptos em diferentes países ${ }^{4}{ }^{14}$. No entanto, C. neoformans var. gattii foi isolado de oco de árvores tropicais no Brasil, como oiti (Moquilea tomentosa), cássia rosa (Cássia grandis), fícus (Fícus microcarpa) e outros, evidenciando outros habitats naturais para esta variedade ${ }^{110}$

A criptococose deve ser considerada no diagnóstico diferencial das infecções oportunistas nos indivíduos imunodeprimidos, sendo fundamental o diagnóstico precoce, para reduzir a morbidade e letalidade desta micose, que na maioria dos casos se apresenta na forma de meningoencefalite.

A ocorrência da criptococose na Região CentroOeste, inclusive em moradores da cidade de Campo Grande, MS, justifica o interesse pelo estudo das fontes de infecção, iniciando pela investigação de $C$. neoformans em excretas de aves em cativeiro, bastante comuns nesta cidade.

\section{MATERIAL E MÉTODOS}

Campo Grande, capital do Estado de Mato Grosso do Sul, faz parte da macro-região do Centro-Oeste brasileiro, basicamente constituído por cerrado, um rico complexo silvestre com exuberante flora, rica fauna, principalmente de espécies de aves, tendo catalogado 714 espécies na região. Neste ambiente urbanizado, com marcada atividade antrópica, escolhemos a área central da cidade, onde foram colhidas 20 amostras de excretas de aves, assim distribuídas: 16 obtidas em 6 lojas de aves, uma obtida em uma escola, uma obtida em um domicílio, e 2 corresponderam a raspados de excretas encontradas em solo de ambiente aberto. Tevese o cuidado de colher material envelhecido e seco, nos poleiros e nas grades das gaiolas e viveiros.

Cada amostra corresponde a uma gaiola ou viveiro, contendo de 4 a 27 aves, agrupadas segundo sua espécie, sem divisão entre machos e fêmeas, representadas por canário belga (Serinus canaria), canário do reino (Corduelis cocullatos), canário da terra (Sicalis flaveola brasiliensis), periquito australiano (Melopsittacus unolulatus), colopsita (Psittacidae), agapore (Psittacidae), mandarim (Psittacidae), pombo rabo de leque (Columba $s p)$, pombo africano (Columba sp), papagaio (Psittacidae). Em duas ocasiões foram observadas espécies diferentes em um mesmo viveiro, agapore com colopsita (amostra $\mathrm{P}-05$ ) e canário com mandarim (amostra P-20), todas essas aves bem adaptadas ao cativeiro.

As amostras foram colhidas em frascos plásticos estéreis e acondicionadas sob refrigeração entre 8 e $12^{\circ} \mathrm{C}$ por 24 horas. O material foi processado em câmara de fluxo laminar (classe 2-B1), homogeneizado em gral com pistilo de porcelana previamente esterilizados. Cerca de $1 \mathrm{~g}$ do material homogeneizado foi suspenso em $50 \mathrm{ml}$ de salina fisiológica estéril contendo $0,4 \mathrm{~g} / \mathrm{l}$ de cloranfenicol. Após agitação em vórtex por 3 minutos, e repouso por 30 minutos à temperatura ambiente, o sobrenadante foi aspirado e $0,1 \mathrm{ml}$ foi semeado em cada placa de petri com meio de sementes de níger (Guizotia abyssinica), contendo 0,2g/l de cloranfenicol e 0,2g/l de amicacina (meio NSA). Foram semeadas 10 placas por amostra, incubadas à temperatura ambiente, entre $26 \mathrm{e}$ $27,5^{\circ} \mathrm{C}$ e observadas diariamente até 7 dias.

Colônias lisas, úmidas, brilhantes e de coloração marromescura foram repicadas na forma de estrias em placa com meio NSA para purificação e obtenção de subcultivos em meio de ágar-Sabouraud $2 \%$ à temperatura ambiente, para realização das provas de identificação. A microscopia foi feita em montagens com $\mathrm{NaOH}$ a $4 \%$, tinta da china (nanquim) e lactofenol azul de algodão. A observação de leveduras de tamanho regulares, globosas, arredondadas, encapsuladas, unibrotantes ou multibrotantes e sem hifas ou pseudo-hifas, conduzia à realização de testes fisiológicos como a produção de urease, termotolerância a $35^{\circ} \mathrm{C}$, sensibilidade à cicloheximida e testes de assimilação de carbono e nitrogênio, realizados em equipamento automatizado, VITEK® (BioMerreux - France).

Para a identificação da variedade foi utilizado o meio canavanina-glicina-azul de bromotimol (CGB) conforme descrito por Kwon-Chung (1984) e a sorotipagem foi realizada pelo kit Crypto Check latron RM 304-K (latron Labs, Tokio, Japão). Este último consiste em teste de aglutinação em lâmina, utilizando anticorpos policlonais, monoespecíficos, correspondentes a fatores séricos.

Foi estimada numericamente a quantidade de propágulos de $C$. neoformans existentes em cada amostra positiva, expressa em unidades formadoras de colônias do fungo por grama de matéria orgânica processada (UFC/g). 


\section{RESULTADOS}

Das 20 amostras analisadas, 10 foram positivas para Cryptococus neoformans var neoformans sorotipo A, correspondentes a 3 locais positivos dentre os 10 ambientes estudados (Tabela 1). A contagem das unidades formadoras de colônias (UFC) de C. neoformans foi realizada em todas as amostras positivas (Tabela 1 ).

\begin{tabular}{|c|c|c|c|c|}
\hline \multirow[b]{2}{*}{ Local } & \multirow[b]{2}{*}{ Gaiola } & \multicolumn{2}{|l|}{ Aves } & \multirow{2}{*}{$\begin{array}{l}\text { Isolamento de } \\
\text { C. neoformans UFC/g }\end{array}$} \\
\hline & & espécie & número & \\
\hline Loja 1 & 1 & periquito australiano & 18 & negativo \\
\hline Loja 2 & 2 & pombo rabo de leque & 3 & negativo \\
\hline \multirow[t]{2}{*}{ Loja 3} & 3 & periquito australiano & 9 & negativo \\
\hline & 4 & canário belga & 12 & negativo \\
\hline \multirow[t]{4}{*}{ Loja 4} & 5 & agapore + colopsita & $12+15$ & 7.000 \\
\hline & 6 & canário & 23 & negativo \\
\hline & 7 & periquito australiano & 27 & 1.000 \\
\hline & 8 & periquito australiano & 27 & 9.000 \\
\hline Loja 5 & 9 & periquito australiano & 18 & negativo \\
\hline Domicílio & 10 & papagaio & 1 & negativo \\
\hline Escola & 11 & $\begin{array}{l}\text { colopsita }+ \\
\text { periquito australiano }\end{array}$ & $2+4$ & 25.000 \\
\hline Pátio & 12 & pardal & 2 & negativo \\
\hline Pátio & 13 & sabiá & 2 & negativo \\
\hline \multirow[t]{7}{*}{ Loja 6} & 14 & periquito australiano & 8 & negativo \\
\hline & 15 & periquito australiano & 15 & 46.200 \\
\hline & 16 & periquito australiano & 30 & 8.100 \\
\hline & 17 & pombo burguesinho & 6 & 11.100 \\
\hline & 18 & pombo burguesinho & 5 & 900 \\
\hline & 19 & pombo rabo de leque & 2 & 1.500 \\
\hline & 20 & canário belga + mandarim & $3+2$ & 6.600 \\
\hline Total & 20 & & 246 & 10 \\
\hline
\end{tabular}

UFC/g Unidades formadoras de colônias por grama de material

\section{DISCUSSÃO}

Trabalhos pioneiros de Emmons, no início da década de 50 , já faziam referência ao acúmulo de excretas secas e envelhecidas de pombos (Columba livia) bem como os solos contaminados com estes excrementos como fontes saprofíticas de $C$. neoformans em ambientes urbanos ${ }^{567}$. Staib (1984) concluiu que a exposição dos homens e animais a excretas dessas aves explica pelo menos em parte a epidemiologia da criptococose $^{27}$. Entretanto, certos aspectos não estão esclarecidos, como a origem de $C$. neoformans nestes excrementos, uma vez que o fungo não é isolado do trato intestinal de aves, onde tem dificuldade de sobreviver à competição microbiana. Por outro lado, há poucos relatos de criptococose em aves ${ }^{9}{ }^{16}$, animais resistentes à invasão pelo fungo devido a sua elevada temperatura corporal. Além disso, já foi relatado o isolamento do fungo de excretas de uma grande variedade de psitacídeos. Essas aves têm o hábito de raspar e fragmentar pedaços de madeira, galhos, e parecem oferecer condições favoráveis de sobrevivência e multiplicação de $C$. neoformans em seus habitats ${ }^{917}$.
Nossos resultados comprovam, pela primeira vez, a contaminação de excretas de aves de cativeiro por C. neoformans var neoformans sorotipo A na cidade de Campo Grande. Foram observadas elevadas concentrações do fungo, até 46.000 propágulos viáveis por grama de material seco, refletindo a existência de fontes ambientais na forma de microfocos deste fungo. Nestes locais o fungo pode ser disperso no ar e, subseqüentemente, inalado. A contaminação observada pode estar relacionada ao grande turn-over de aves nas gaiolas, a forma de limpeza das gaiolas ou viveiros com a mesma ferramenta, e a grande disponibilidade de sementes de níger, painço, alpiste e girassol nos locais, que podem servir de substrato para o crescimento do fungo. Estudos conduzidos por Mitchel \& Perfect relataram que é grande o número de pessoas com anticorpos circulantes após exposição e manejo de aves e habitats contaminados. Não desenvolvem, porém, a doença, reafirmando a idéia de que a variedade neoformans é de baixa patogenicidade e comporta-se essencialmente como oportunista ${ }^{16}$. 
Swinne et al isolaram $C$. neoformans var neoformans da poeira doméstica das amostras oriundas das residências dos pacientes com criptococose associada a AIDS e em contato com aves em $35 \%$ das residências analisadas no Bujumbura, Burundi (África Central) ${ }^{29}{ }^{30}$. Passoni et al. encontraram em torno de $15 \%$ de positividade de isolamento de $C$. neoformans var neoformans da poeira doméstica no Rio de Janeiro, valorizando o achado do fungo em excretas de aves em cativeiro, como canários, periquitos e outros psitacídeos. Foi demonstrada uma correlação significativa entre a existência de aves cativas no ambiente domiciliar e a probabilidade de contaminação destas residências por C. neoformans. Conseqüentemente, pacientes com AIDS apresentavam risco aumentado em adquirir a doença quando residentes em domicilio positivo para o fungo ${ }^{1718}$.

Entre outubro de 1994 e fevereiro de 1998 foram registrados 23 casos de meningoencefalite criptococócica no Hospital Universitário da UFMS, na cidade de Campo
Grande, centro de referência do estado para meningites. Destes, 18 pacientes estavam com AIDS e 5 não tinham condição predisponente evidente. Vinte residiam em Mato Grosso do Sul, sendo 14 naturais deste estado e 12 procedentes de Campo Grande. A absoluta maioria era de área urbana. C. neoformans var gattii ocorreu em dois casos e os demais 21 pacientes apresentavam doença causada por C. neoformans var neoformans sorotipo $A^{20}$. $O$ encontro deste mesmo agente e sorotipo em excretas de fezes de aves de cativeiro correlacionam com os dados da casuística local da micose humana.

Estes achados trazem à luz a questão de minimizar o risco de exposição a microfocos de $C$. neoformans var neoformans em locais de circulação pública e em domicílios de pacientes imunodeprimidos através da vigilância das condições de higiene e limpeza de excretas, aeração, iluminação e ventilação adequadas, bem como monitorização de locais de risco.

\section{REFERÊNCIAS BIBLIOGRÁFICAS}

1. Cavalcanti MAS. Criptococose e seu agente no Meio Norte, Estados de Piauí e Maranhão, Brasil. Tese de Doutorado, Universidade Federal do Piaui, Instituto Oswaldo Cruz Fundação Instituto Oswaldo Cruz, Rio de Janeiro, RJ, 1997.

2. Collins HL, Bancroft GJ. Encapsulation of Cryptococcus neoformans impairs antigen-specific T-cell responses. Infection and Immunity 59: 3883-3888, 1991.

3. Ellis DH, Pfeiffer TJ. Ecology, life and infectious propagule of Cryptococcus neoformans. Lancet 336: 923-925, 1990.

4. Ellis DH, Pfeiffer TJ. Natural habitat of Cryptococcus neoformans var. gattii. Journal of Clinical Microbiology 28: 1642-1644, 1990.

5. Emmons CW. Saprophytic sources of Cryptococcus neoformans associated with the pigeon (Columba livia). American Journal of Hygiene 62: 227-232, 1955.

6. Emmons CW. Prevalence of Cryptococcus neoformans in pigeon habitats. Public Health Reports 75: 362-364, 1960.

7. Emmons CW. Natural occurrence of opportunistic fungi. Laboratory Investigation Baltimore 11: 1026-1032, 1962.

8. Kwon-Chung KJ, Bennett JE. Epidemiologic differences between the varieties of Cryptococcus neoformans. American Journal of Epidemiology 120:123-130, 1984.

9. Kwon-Chung KJ, Bennett JE. Cryptococcosis In: Camilo-Coura L, Febiger (eds) Medical Mycology. Philadelphia, p. 397-446, 1992.

10. Lazéra MS, Cavalcanti MAS, Londero AT, Trilles L, Nishikawa MM Wanke B. Possible primary ecological niche of Cryptococcus neoformans. Medical Mycology 38: 379-383, 2000.

11. Lazéra MS, Cavalcanti MAS, Trilles L, Nishikawa MM, Wanke B. Cryptococcus neoformans var. gattii - evidence for a natural habitat related to decaying wood in a pottery tree hollow. Medical Mycology 36: 119-122, 1998.

12. Lazéra MS, Pires FDA, Camillo-Coura L, Nishikawa MM, Bezerra CCF, Trilles L, Wanke B. Natural habitat of Cryptococcus neoformans var. neoformans in decaying wood forming hollows in living trees. Journal of Medical and Veterinary Mycology $34: 127-131,1996$
13. Lazéra MS, Wanke B, Nishikawa MM. Isolation of both varieties of Cryptococcus neoformans from saprophytic sources in the city of Rio de Janeiro, Brazil. Journal of Medical and Veterinary Mycology 31:449-454, 1993.

14. Laurenson IF, Lalloo DG, Naraqi S, Seaton RA, Trevett AJ, Matura A, Kevau IH. Cryptococcus neoformans in Papua New Guinea: a common pathogen but an elusive source. Journal of Medical and Veterinary Mycology 35: 437-440, 1997.

15. Levitz SM. The ecology of Cryptococcus neoformans and the epidemiology of cryptococcosis. Review of Infectious Diseases 13:1163-1169, 1991.

16. Mitchel TG, Perfect Jr. Cryptococcosis in the era of AIDS-100 years after the discovery of Cryptococcus neoformans. Clinical Microbiology Reviews 8: 515-548, 1995.

17. Passoni LFC. Wood, animals and human beings as reservoir for human Cryptococcus neoformans infection. Revista Iberoamericana de Micologia 16: 77-81, 1999.

18. Passoni LFC, Wanke B, Nishikawa MM, Lazéra MS. Cryptococcus neoformans isolated from human dwellings in Rio de Janeiro, Brazil: An analysis of domestic environment of AIDS patients with and without cryptococcosis. Medical Mycology 36: 305-311, 1998.

19. Pfeiffer TJ, Ellis D. Environmental isolation of Cryptococcus neoformans var. gattii from Eucalyptus tireticornis. Journal of Medical and Veterinary Mycology 30: 407-408, 1992.

20. Reis MAC. Estudo clínico-epidemiológico de 23 casos de meningoencefalite criptocócica internados no Hospital da Universidade Federal de Mato Grosso do Sul no período de outubro de 1994 a fevereiro de 1998. Dissertação de conclusão do curso de Medicina. Universidade Federal de Mato Grosso do Sul, Campo Grande, MS, 1998.

21. Restrepo A, Baumgardner DJ, Bagagli E, Cooper Jr CR, McGinnis MR, Lazéra MS, Barbosa FH, Bosco SMG, Camargo ZP, Coelho KIR, Fortes ST, Franco M, Montenegro MR, Sano A, Wanke B. Clues to the presence of pathogenic fungi in certain environments. Medical Mycology 38 (supl. 1): 67-77, 2000. 
22. Rippon JW. Medical Mycology. In: The pathogenic fungi and pathogenic actinomycetes. W.B. Saunders, New York, p. 582-609, 1988.

23. Rosenbaum R, Gonçalves AJR. Clinical epidemiological study of 171 cases cryptococcosis. Clinical Infectious Diseases 18:369380, 1994

24. Sorrell TC, Ellis DH. Ecology of Cryptococcus neoformans. Revista Iberoamericana de Micologia 14: 42-43, 1997.

25. Staib F. Saprophytic life of Cryptococcus neoformans. Annales de la Société Belge de Médicine Tropicale 44: 611-618, 1964.

26. Staib F. Cryptococcus neoformans and cryptococcosis. Peculiarities and challenge: opening lecture of second International Conference on Cryptococcus and cryptococcosis. Milan (Italy), September 19-23, 1993. Journal Mycology Medical 4:56-60, 1994.

27. Staib F, Schuultz-Dieterjch J. Cryptococcus neoformans in fecal matter of bird's kept in cages - control of Cryptococcus neoformans habitats. Zentralblatt für Bakteriologie und Parsitenkunde 174: 79-186, 1984.

28. Swinne D, Bauverns L, Desmet P. More information about the natural habitat of Cryptococcus neoformans. Newsletter International Society for Human and Animal Micology 60:4, 1992.

29. Swinne D, Deppner M, Laroche R, Floch JJ, Kadende P. Isolation of Cryptococcus neoformans from houses of AIDS-associated cryptococcosis patients in Bujumbura (Burundi). AIDS 3: 389390, 1989.

30. Swinne D, Taelman H, Batungwanayo J, Bigirankana A, Bogaerts J. Contribuition a l'etude de l'ecologie de Cryptococcus neoformans em Afrique centrale. Médicine Tropicale 54: 53-55, 1994.

31. Wicles BL, Mayorga ME, Edman U, Edman JC. Dimorphism and haploid fruiting in Cryptococcus neoformans: Association with the a-mating type. Proceeding of the National Academy of Science 93: 7327-7331, 1996. 